\title{
Platelet-rich concentrates (L-PRF, PRP) in tissue regeneration: Control of apoptosis and interactions with regenerative cells
}

\author{
Alessandro Crisci ${ }^{1,2,3 *}$, Ugo De Crescenzo ${ }^{4}$ and Michela Crisci ${ }^{5}$ \\ ${ }^{1}$ Unit of Dermosurgery Cutaneous Transplantations and Hard-to-Heal Wound, "Villa Fiorita” Private Hospital, Aversa CE, Italy \\ ${ }^{2}$ Institute for the Studies and Care of Diabetcs, “Abetaia”, Casagiove CE, Italy \\ ${ }^{3}$ School of Medicine, University of Salerno Italy, Fisciano SA, Italy \\ ${ }^{4}$ Anesthesia and Resuscitation, "Villa Fiorita" Private Hospital, Aversa CE, Italy \\ ${ }^{5}$ Faculty of Medicine and Surgery, Vasile Goldis Western University of Arad, 310025 Arad, Romania
}

\begin{abstract}
Platelet-derived Growth Factors (GFs) are biologically active peptides that enhance tissue repair mechanisms such as angiogenesis, extracellular matrix remodeling, and cellular effects as stem cells recruitment, chemotaxis, cell proliferation, and differentiation. Platelet-rich concentrates (L-PRF, PRP) play a critical role in tissue repair and regeneration. They regulate fundamental mechanisms involved in the healing process including cellular migration, proliferation, and angiogenesis. Control of apoptosis/cell survival and interaction with progenitor cells, which are clinically relevant but poorly understood aspects of platelets in tissue repair, will be highlighted in this review. Gaining deeper insight into the less well-characterized molecular mechanisms is necessary to develop new therapeutic platelet-based options.
\end{abstract}

\section{Introduction}

The platelet-rich fibrin (PRF) is a platelet concentrate of the latest generation, consisting of P-PRF (pure platelet-rich fibrin) and L-PRF (leukocyte-containing platelet-rich fibrin). Fibrin gels -PRP (plateletrich plasma) and PRF- are optimal as scaffolds for tissue engineering for various reasons. The most important reason is the compatibility with the cell life of fibrin, which is different according to different completely biodegradable natural substances, and it facilitates the transition to a new extracellular reticule. In addition to mediating primary hemostasis and thrombosis, platelets play an important role in repairing and regenerating tissues. They regulate the main mechanisms involved in the healing process, including cell migration, proliferation, and angiogenesis. The PRF clot forms a robust fibrin grid with a complex 3-dimensional architecture. By quantifying the presence of growth factors [Growth factor derived from platelets (PDGF-BB), transforming growth factor $\beta 1$ (TGF-1 $\beta$, and insulin-like growth factor-1 (IGF1)] in PRF, enzyme-linked immunosorbent assay analyses revealed that slow fibrin polymerization during PRF processing leads to the intrinsic constitution of platelets of cytokines and glycan chains in fibrin reticulum [1]. P-PRF and L-PRF are biological materials with solid fibrin and/or containing leukocytes. All the platelets contained in blood samples taken are activated and integrated into the coagulum fibrin reticulum. Approximately $97 \%$ of platelets and $50 \%$ of leukocytes are concentrated in the PRF clot showing a specific 3-dimensional distribution.

Growth factors released by L-PRF platelets, particularly TGF- $1 \beta$, induce and control the proliferation and migration of other types of cells involved in tissue repair such as smooth muscle cells (SMCs) and mesenchymal stem cells (MSCs).

The control of apoptosis and cell survival and interaction with progenitor cells are clinically important; however, the aspects of platelets in tissue repair are poorly understood, which are highlighted in this review.

The advantages in the production/use of the PRF's compared to the PRP are:

- None biochemical blood manipulation;

- Simplified and cheaper manufacture;

- Use of bovine thrombin and anticoagulants are not requested;

- Positive healing thanks to a slow polymerization:

- More efficient cellular migration and proliferation;

- PRF have a good effect on immune system;

- PRF helps haemostasis [1].

${ }^{\star}$ Correspondence to: Alessandro Crisci, Unit of Dermosurgery Cutaneous Transplantations and Hard-to-Heal Wound, "Villa Fiorita" Private Hospital, 81031 Aversa CE, Italy, E-mail: alessandrocrisci@libero.it

Key words: blood derivatives, growth factors, leucocyte and platelets rich fibrin, plasma rich platelets, stem cells

Received: August 25, 2018; Accepted: September 05, 2018; Published: September 07, 2018 


\section{Platelets: As healers of damaged tissues}

Platelets have been used to treat patients with thrombosis, thrombocytopenia, or bleeding events to restore hemostasis. However, platelets also function as circulating cell receptors that provide a unique link between immune responses and tissue repair. Wound repair is inseparably associated with inflammation and requires a finely synchronized combination of the mechanisms regulating cell migration, the organization and remodeling of the extracellular matrix, cell proliferation, differentiation and angiogenesis, and neovascularization [2]. Platelets have been recognized to be involved in all of these cell events, which are examined elsewhere [3].

This review provides an update on the intensively investigated role of platelets in tissue regeneration and highlights clinically relevant yet incompletely characterized mechanisms, namely interactions with progenitor cells and apoptosis control and cell survival. Attention to platelet-rich plasma and PRF has increased progressively in wound healing and improved wound healing [4]. The clinical and experimental data clearly indicate that platelets are fundamentally involved in the repair and regeneration of damaged tissues and the preservation of organ function. During tissue injuries, for example, injuries caused by trauma or human tissues ischemia such as in myocardial infarction or cerebral, the coagulation system and the immune responses are activated very early, starting the wound healing process. Platelets are the first cells that accumulate at the sites of the lesion and, upon activation, release a multitude of biologically active mediators in their environment. Various cytokines, chemokines, growth factors, including growth factor derived from stroma 1 (SDF-1) [5] and the hepatocyte growth factor (HGF) [6] are secreted by platelets. Platelet-derived mediators induce and modulate the late activation of fibroblasts and the recruitment of leukocytes, the first neutrophils, followed by macrophages, with consequent elimination of dead cells and cellular debris [2]. Furthermore, the factors released by platelets induce and control the proliferation and migration of other types of cells involved in tissue repair, such as SMCs and MSCs [7]. Angiogenesis in damaged tissues, another key mechanism for recovery of tissue function, is also substantially regulated by platelets because of the release of a multitude of proangiogenic and antiangiogenic mediators from activated platelets 3 (Figure 1). Today, platelets and their secreted products can be used successfully as feasible therapeutic tools, facilitating the repair of damaged tissues and organs. For example, because autologous platelets also release recombinant growth factors from platelets [8], they can improve the healing of chronic diabetic ulcers of the lower limbs [9]. Furthermore, regeneration of skin wounds [9], retina, and bones has been reported. However, the treatment of surgical lesions with plateletrich plasma (PRP) also produced controversial results in clinical trials [10].

In vitro behavior of a membrane and $\mathrm{L}-\mathrm{PRF}$ and Gel P-PRP (Platelet Rich Plasma pure) (PRFG-endorest) were compared through the evaluation of the slow release of growth factors and matrix molecules. These two families of concentrates were placed in a culture medium for 7 days, and the versions slowed of 3 factors of key growth. (TGF- $\beta 1$, PDGF-AB, VEGF) and 3 coagulation proteins and matrix (TSP-1 [thrombospondin-1]) (Fn) (vitronectin) were quantified experimentally seven times (at 20, 1h, 4h, 24h, 72h, 120h, 168h) [1-9].

These studies revealed that the products have two very different profiles: the membrane L- PRF remained solid and intact after 7 days (up to 28 days) and continuously releases a large amount of growth factors, a significant part of it is produced by cells inside of the membrane. In contrast the gel P-PRP clarified releases most of the growth factors in the early hours and is fully dissolved after 3 days.

L-PRF was also used in diabetic foot ulcer osteomyelitis allowing recovery from this serious disease [11]. L-PRF is a new step in the therapeutic concept of platelet gel with a simplified design and small artificial biochemical changes. Unlike other platelet concentrates, this

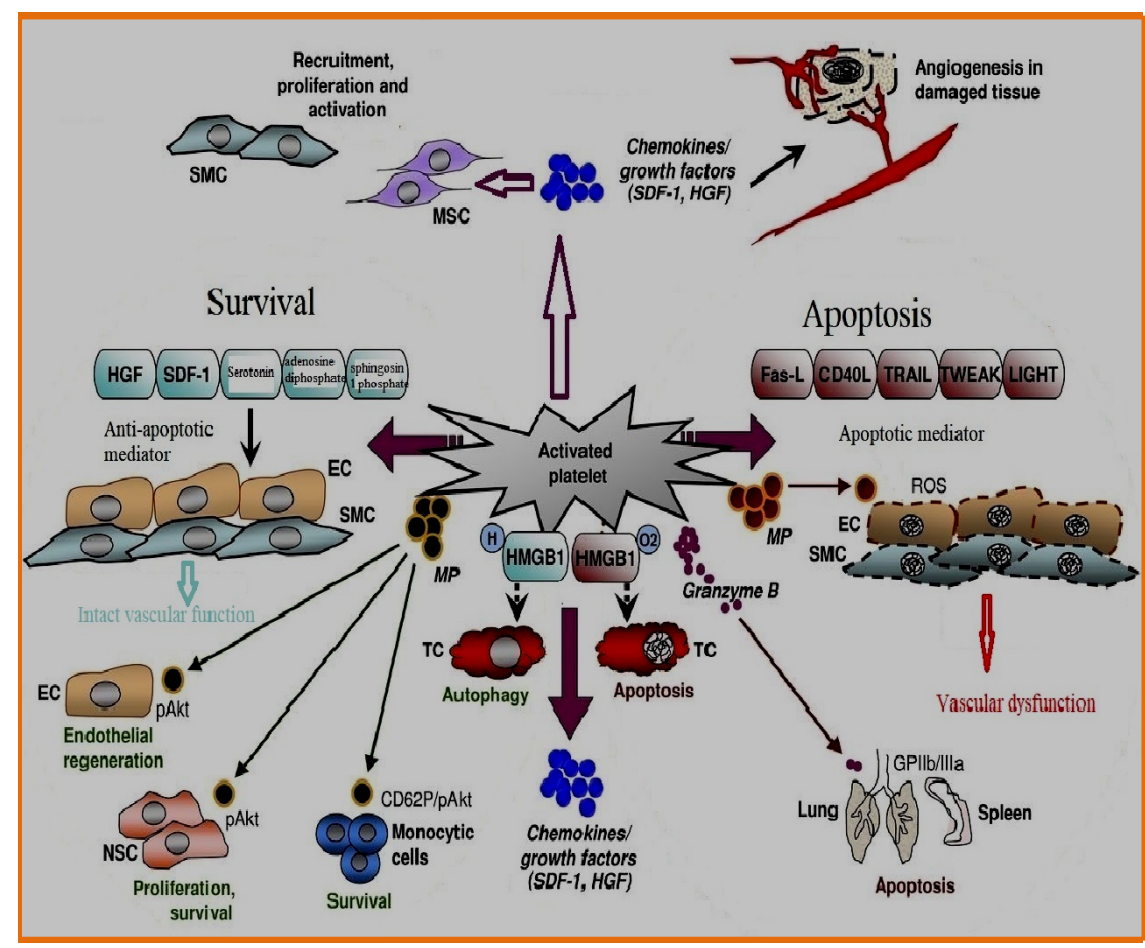

Figure 1. Mechanisms regulating tissue repair mediated by platelets 
technique does not require anticoagulants, thrombin, or any other gelling agent, which requires centrifuge of blood without additives $[3,4]$. Although leukocyte platelets and cytokines play an important role in the biology of this biomaterial, the supporting fibrin matrix is certainly the decisive factor in the real therapeutic potential of L-PRF. Within a few minutes, the absence of an anticoagulant allows the activation of most of the platelets contained in the sample to trigger the coagulation cascade.

PRP has recently emerged as a new matrix to improve the survival of transplanted fat. PRP, derived from whole blood through doubleturn centrifugation, contains multiple growth factors and adhesion molecules in the $\alpha$-granules. PRP is believed to be safer and more practical in adjunctive clinical therapy than other recombinant growth factors or stem cell therapies. PRP is shown to be effective in bone regeneration, wound healing, and improvement of musculoskeletal sport injuries [12]. The scope of PRP therapy has been extended to soft tissue augmentation by combining PRP with fat grafting. Although some positive clinical results have been reported [12], trials supporting the application of PRP combined with fat grafts in soft tissue augmentation remain limited, since only a few basic studies and preclinical studies on small animals have been conducted. In ten patients with chronic liver disease, platelet transfusion improved distinct parameters of liver function, although adverse events related to platelet transfusion were observed. An important observation in understanding the functions of platelets and their limitations in terms of tissue repair was made in the field of hepatic pathophysiology. In a mouse model, Lesurtel, et al. [13] identified platelet-derived serotonin as the key factor for liver regeneration. Thrombocytopenia and decreased platelet activity in mice substantially abolished cell proliferation in the liver. In contrast, thrombopoietin-induced thrombocytosis caused a strong platelet accumulation in the sinusoids of the liver and the induction of hepatocyte proliferation shortly after hepatectomy in mice [14]. Furthermore, platelets have been shown to be involved in the postnatal occlusion of the ductus arteriosus and vessel remodeling [15]. Adhesion and malfunctioning platelet aggregation and defective platelet biogenesis have been associated with postnatal occlusion of ductus in newborn mice. Furthermore, premature neonates with thrombocytopenia showed an increased risk of a persistent open duct. The impact of abnormal platelet function on tissue repair has also been studied in atypical uremic syndrome with complement factor derived from platelet dysfunction. In addition to its complementary regulatory protein function, the $\mathrm{H}$ factor exerts an anti-inflammatory activity and its mutations contribute substantially to hemolytic uremic syndrome and damage to the glomerular membrane, resulting in type II proliferative membranous glomerulonephritis. Thus, evidence indicates that platelets or platelet-derived factors play a key role in determining tissue balance between repair and tissue damage and can therefore be successfully used in regenerative care. However, the underlying molecular mechanisms involved in platelet-mediated tissue repair are poorly characterized and further experimental and clinical studies are required to define the specific targets for future therapeutic interventions.

\section{Interaction and regeneration of platelet cells and progenitor cells}

Activated platelets release a full range of chemokines [16] and promote the recruitment, adherence, and proliferation of adult stem cells, including CD34-positive progenitor cells, MSCs, SMC progenitors, and endothelial progenitors [7] (Figure 1). The multipotency of these types of stem cells and their ability to increase vascular tissue repair is attributed to paracrine mechanisms [17] and make them promise in regenerative medicine. Furthermore, tissue damage itself generates strong chemoattractive signals for stem cells, providing the basis for their regenerative activity. Platelets regulate the recruitment of adult stem cells to injured cells; this can, therefore, be an important mechanism in regenerative cellular responses. After a myocardial infarction, intramuscular, intracoronary, or intravenous MSC transplantation, cells migrate to the injured heart, significantly restoring cardiac function [18]. Various clinical studies have confirmed the beneficial effects of MSCs after myocardial damage even if the clinical benefit was occasionally moderate. HGF is a growth factor known to be produced after myocardial ischemia, as investigated in animals and humans. With its antiapoptotic, proangiogenetic, and immunosuppressive activity [19], HGF exerts its cardioprotective properties. Platelets are also released after the activation of HGF [6] and have been described to promote the uptake of MSCs into human artery endothelial cells [7]. Thus, the modulation of HGF-mediated migration of MSC to apoptotic tissue cells by platelets is probable and can become a powerful therapeutic tool to improve cardiac function after myocardial infarction. As with HGF, another major mediator involved in stem cell trafficking, SDF-1, is also regulated after myocardial ischemia. SDF-1/CXCR4 has been shown to induce bone marrow progenitor recruitment into bones after intravenous injection of cells into a mouse model. In clinical studies, CD34-positive progenitor cells have been critically reported to be involved in myocardial repair and regeneration, contributing to the preservation of cardiac function. Furthermore, compared with control mice, the injection of recombinant SDF-1 into the left ventricular cavity of test mice before coronary occlusion significantly decreased the infarct size. To improve the effectiveness of SDF-1-mediated cardioprotection, we synthesized a bifunctional protein consisting of an SDF-1 domain and a glycoprotein (GP) VI domain with a high CXCR4-binding affinity as well as extracellular matrix proteins that become exposed after tissue lesions [20]. After experimental myocardial infarction, the administration of SDF1-GPVI exerted significant cardioprotective effects, promoting CXCR4 positive migration of progenitors from the bone marrow, strengthening endothelial differentiation of the, preserving cell survival, and revealing proangiogenic effects. Platelets influence these progenitor cells mediated by SDF-1 activity. SDF-1 secreted by CD34-positive activated platelets supports the recruitment of progenitor cells into arterial thrombus and differentiates the cells into in vivo endothelial progenitor cells. In patients with myocardial infarction, SDF-1 derived from platelets control a number of circulating progenitor cells and is associated with the restoration of left ventricular function and improves prognosis. Furthermore, the formation of coregulated circulating CD34-positive progenitor cells/platelets has been described in patients with acute coronary syndromes, associated with a significant decrease in myocardial infarct size, improving the function of the left ventricle, as seen with cardiac magnetic resonance imaging at a 3-month follow up [21]. The differentiation of CD34-positive platelets in mature foam cells and in endothelial cells has been described in a vitro co-culture system, which may be particularly relevant for the development of atherosclerotic vascular lesions.

\section{The regulatory platelets of apoptosis and cell survival: Tissue regeneration mechanisms}

Apoptosis is a process of cell death that is performed exactly to limit tissue damage and is generally associated with immune tolerance. The regulation of the balance between apoptosis and cell survival, which is 
important for the fate of damaged tissues, is controlled by platelets [22] (Figure 1). The induction of apoptosis is regulated by different cellular signals, which can be extracellular and intracellular. An important extracellular apoptotic pathway involves death receptors, which are the members of the tumor necrosis factor (TNF) receptor family. TNF- $\alpha$ is one of the main cytokines that regulates apoptosis. Although the presence of TNF- $\alpha$ in platelets is not confirmed, platelets store and secrete various ligands linked to TNF- $\alpha$, such as Fas-L, CD40L, TRAIL, TWEAK, and LIGHT [23], which regulate apoptosis through paracrine signals. The incubation of endothelial cells and SMCs with microparticles derived from platelets of patients with sepsis resulted in a strong induction of apoptosis in cells, due to the production of reactive oxygen species (ROS), suggesting a central mechanism in the pathogenesis of septic vascular dysfunction. The role of platelet microparticles has been demonstrated in the phosphorylation and activation of Akt (pAkt), a serine threonine kinase that inactivates the pro-apoptotic BAD part of lymphomas [2] (associated B lymphoma 2 cell, a promoter of cell death), exercising an antiapoptotic activity in THP-1 cells, a cell line of human monocytic leukemia, in a P selectindependent manner [22]. Some types of platelet microparticles are induced to differentiate into monocytes through intracellular calcium flows and the release of the complement factor C5a and TNF- $\alpha$. Sharron, et al. [24] demonstrated that platelets from mice with sepsis induced by apoptosis in CD4-positive mice underwent through a mechanism independent of platelet microparticles. In this study, apoptosis was mediated by the protease of serine granzyme $\mathrm{B}$, which is regulated by megakaryocytes of mice with sepsis. Later, the authors demonstrated that platelet-induced granzyme B apoptosis occurred in the spleen and in the lung depending on the direct contacts of the cells and on the correct cellular function of GPIIb/IIIa [24] (Figure 1). Platelets can perform antiapoptotic mechanisms, shifting balance to cell survival and tissue repair. In neural stem cells (CNS), platelet microparticles caused the phosphorylation of Akt, associated with cell proliferation, and survival and differentiation of neuronal cells [25]. Akt-mediated phosphorylation of platelet microparticles has also been observed in endothelial cells and improved endothelial regeneration occurred after the injection of angiogenic cells. Furthermore, platelets secrete, at activation, mediators with antiapoptotic activity, such as HGF, SDF1 , serotonin, adenosine diphosphate, and sphingosine-1 phosphate (Figure 1), favoring survival signals for vascular endothelial cells and SMCs at the sites of vascular lesions. HMGB1, a nuclear protein passively released by necrotic cells during tissue injury or actively secreted by innate immune cells, has been identified as a warning signal that activates an immune response and regulates the cell death and survival, as demonstrated for cancer cells, depending on the status of HMGB1 redox. Platelets contain endogenous HMGB1, which is exported to the cell surface after activation, and this makes HMGB1 a candidate for regulating platelet mortality/survival balance. The type of target cells and the regional distribution and the intensity of expression of the surface receptors can define the result of the proapoptotic and antiapoptotic functions of platelets.

\section{Conclusions}

Apoptosis is a mechanism of cell death together with necrosis. Most cells have the ability to self-destruct through the activation of a death program, to which only specialized cells they subtract, such as those of the heart muscle and nervous tissue.

Unlike necrosis, apoptosis plays a role evolutionary very important, as it regulates all existence of the organism starting from the embryonic development. Platelets can perform antiapoptotic mechanisms, shifting balance to cell survival and tissue repair. They secrete mediators with antiapopethic activity (HGF, SDF-1 etc.) favoring the signals of cellular survival.

Platelets regulate the recruitment of adult stem cells into wounded cells; this can therefore be an important mechanism in regenerative cellular responses. Platelets are also released after HGF activation and have been described to promote the uptake of MSC into human artery endothelial cells.

In summary, L-PRF may offer advantages over L-PRP comprising overall higher amounts of released TGF- $\beta 1$, a sustained, long-term release of investigated growth factors, and stronger induction of cell migration.

Other experimental and clinical studies must be conducted to provide a better understanding of the crosstalk between the platelets and the mechanisms that control tissue repair, characterized by processes such as the recruitment of cells with regenerative potential and the regulation of apoptosis/cell survival.

The use of biological materials and blood fractions is also a legal problem regarding analytic tests, use of products not for transfusion use and of coordination among blood transfusion centres, laboratories, tissue banks, industry and patient. Although from a legal point of view a doctor being authorized for intravenous punctures is authorized also to the levy. Furthermore, by regulation Community, then with law valid throughout Europe the L-PRF not having added substances it is not a blood derivative but falls within the case of re-grafting of autologous cells $[1,9]$.

These new insights will help us find better therapeutic options based on platelets to facilitate the repair and regeneration of damaged tissues and organs.

Platelets are cell mediators that orchestrate clinically relevant, but not yet known, mechanisms of tissue repair. They release cytokines, chemokines, and growth factors such as SDF-1 and HGF that control the recruitment, proliferation, and activation of SMCs, MSCs, and other types of cells that are chiefly involved in wound healing. Platelets also regulate angiogenesis in damaged tissues. The recruitment of progenitor cells, including MSCs, SMCs, endothelial progenitors, and CD34positive progenitors, is also influenced by platelets, promoting wound repair at least in part due to paracrine mechanisms. Furthermore, platelets are able to modulate the balance between apoptosis and cell survival, which determines the pathophysiology of damaged tissues. They can release proapoptotic mediators (Fas L, CD40L, TRAIL, TWEAK, and LIGHT) and antiapoptotic agents (HGF, SDF1, serotonin, adenosine diphosphate, and sphingosine-1-phosphate). Furthermore, microparticles derived from platelets (MPs) can regulate apoptosis in endothelial cells (ECs) and SMCs, as well as provide survival signals to monocytes and endothelial and neural stem cells. Granzyme B is a mediator of platelet-induced apoptosis in the spleen and lungs. HMGB1, a danger signal that is exported to the cell surface from platelets to activation, regulates apoptosis and autophagy in tumor cells (TCs) depending on its redox state. Thus, platelets control complex tissue repair mechanisms.

\section{References}

1. Crisci A (2015) Le membrane L-PRF utili in chirurgia. J Plast Dermatol 2: 75-90.

2. Gurtner GC, Werner S, Barrandon Y, Longaker MT (2008) Wound repair and regeneration. Nature 453: 314-321. [Crossref]

3. Stellos K, Kopf S, Paul A, Marquardt JU, Gawaz M, et al (2010) Platelets in regeneration. Semin Thromb Hemost 36: 175-184. [Crossref] 
4. Lacci KM, Dardik A (2010) Platelet-rich plasma: support for its use in wound healing. Yale J Biol Med 83: 1-9. [Crossref]

5. Massberg S, Konrad I, Schürzinger K, Lorenz M, Schneider S, et al. (2006) Platelets secrete stromal cell-derived factor 1alpha and recruit bone marrow-derived progenitor cells to arterial thrombi in vivo. $J$ Exp Med 203: 1221-1233. [Crossref]

6. Nakamura T, Teramoto H, Ichihara A (1986) Purification and characterization of a growth factor from rat platelets for mature parenchymal hepatocytes in primary cultures. Proc Natl Acad Sci USA 83: 6489-6493. [Crossref]

7. Langer HF, Stellos K, Steingen C, Froihofer A, Schönberger T, et al. (2009) Platelet derived bFGF mediates vascular integrative mechanisms of mesenchymal stem cells in vitro. J Mol Cell Cardiol 47: 315-325. [Crossref]

8. Margolis DJ, Kantor J, Santanna J, Strom BL, Berlin JA (2001) Effectiveness of platelet releasate for the treatment of diabetic neuropathic foot ulcers. Diabetes Care 24: 483488. [Crossref]

9. Crisci A, Placido F, Crisci M, Bosco A (2015) A new instrument aid of plastic surgeon: membranes L-PRF (Pletelet-Rich-Fibrin). Update Plast Surg 3: 162-172

10. Carreon LY, Glassman SD, Anekstein Y, Puno RM (2005) Platelet gel (AGF) fails to increase fusion rates in instrumented posterolateral fusions. Spine (Phila Pa 1976) 30: E243-246. [Crossref]

11. Crisci A, Marotta G, Licito A, Serra E, Benincasa G, et al. (2018) Use of leukocyte platelet (L-PRF) rich fibrin in diabetic foot ulcer with osteomyelitis (three clinical cases report). Diseases 6: 30. [Crossref]

12. Liao HT, Marra KG, Rubin JP (2014) Application of platelet-rich plasma and plateletrich fibrin in fat grafting: basic science and literature review. Tissue Eng Part B Rev 20: 267-276. [Crossref]

13. Lesurtel M, Graf R, Aleil B, Walther DJ, Tian Y, et al. (2006) Platelet-derived serotonin mediates liver regeneration. Science 312: 104-107. [Crossref]

14. Murata S, Ohkohchi N, Matsuo R, Ikeda O, Myronovych A, et al. (2007) Platelets promote liver regeneration in early period after hepatectomy in mice. World J Surg 31: 808-816. [Crossref]
15. Echtler K, Stark K, Lorenz M, Kerstan S, Walch A, et al. (2010) Platelets contribute to postnatal occlusion of the ductus arteriosus. Nat Med 16: 75-82. [Crossref]

16. von Hundelshausen P, Petersen F, Brandt E (2007) Platelet-derived chemokines in vascular biology. Thromb Haemost 97: 704-713. [Crossref]

17. Gnecchi M, Zhang Z, Ni A, Dzau VJ (2008) Paracrine mechanisms in adult stem cel signaling and therapy. Circ Res 103: 1204-1219. [Crossref]

18. Gnecchi M, He H, Liang OD, Melo LG, Morello F, et al. (2005) Paracrine action accounts for marked protection of ischemic heart by Akt-modified mesenchymal stem cells. Nat Med 11: 367-368. [Crossref]

19. Okunishi K, Dohi M, Nakagome K, Tanaka R, Mizuno S, et al. (2005) A novel role of hepatocyte growth factor as an immune regulator through suppressing dendritic cell function. J Immunol 175: 4745-4753. [Crossref]

20. Ziegler M, Elvers M, Baumer Y, Leder C, Ochmann C, et al. (2012) The bispecific SDF1-GPVI fusion protein preserves myocardial function after transient ischemia in mice. Circulation 125: 685-696. [Crossref]

21. Stellos K, Bigalke B, Borst O, Pfaff F, Elskamp A, et al. (2013) Circulating plateletprogenitor cell coaggregate formation is increased in patients with acute coronary syndromes and augments recruitment of $\mathrm{CD} 34+$ cells in the ischaemic microcirculation. Eur Heart J 34: 2548-2556. [Crossref]

22. Vasina EM, Cauwenberghs S, Staudt M, Feijge MA, Weber C, et al. (2013) Aging- and activation-induced platelet microparticles suppress apoptosis in monocytic cells and differentially signal to proinflammatory mediator release. Am J Blood Res 3: 107-123. [Crossref]

23. Meyer T, Amaya M, Desai H, Robles-Carrillo L, Hatfield M, et al. (2010) Human platelets contain and release TWEAK. Platelets 21: 571-574. [Crossref]

24. Sharron M, Hoptay CE, Wiles AA, Garvin LM, Geha M, et al. (2012) Platelets induce apoptosis during sepsis in a contactdependent manner that is inhibited by GPIIb/IIIa blockade. PLoS One 7: e41549. [Crossref]

25. Hayon Y, Dashevsky O, Shai E, Varon D, Leker RR (2012) Platelet microparticles promote neural stem cell proliferation, survival and differentiation. J Mol Neurosci 47: 659-665. [Crossref]

Copyright: (C2018 Crisci A. This is an open-access article distributed under the terms of the Creative Commons Attribution License, which permits unrestricted use, distribution, and reproduction in any medium, provided the original author and source are credited. 